\title{
Current collapse in tunneling transport through benzene
}

\author{
M. H. Hettler, ${ }^{1}$ W. Wenzel, ${ }^{1}$ M. R. Wegewijs, ${ }^{2}$ and H. Schoeller ${ }^{2}$ \\ ${ }^{1}$ Forschungszentrum Karlsruhe, Institut für Nanotechnologie, 76021 Karlsruhe, Germany \\ ${ }^{2}$ Institut für Theoretische Physik A, RWTH Aachen, 52056 Aachen, Germany
}

(Dated: October 23, 2018)

\begin{abstract}
We investigate the electrical transport through a system of benzene coupled to metal electrodes by electron tunneling. Using electronic structure calculations, a semi-quantitative model for the $\pi$ electrons of the benzene is derived that includes general two-body interactions. After exact diagonalization of the benzene model the transport is computed using perturbation theory for weak electrode-benzene coupling (golden rule approximation). We include the effect of an applied electric field on the molecular states, as well as radiative relaxation. We predict a current collapse and strong negative differential conductance due to a "blocking" state when the electrode is coupled to the para-position of benzene. In contrast, for coupling to the meta-position, a series of steps in the $I-V$ curve is found.
\end{abstract}

PACS numbers: 73.63.-b, 73.23.Hk, 73.22.-f

Introduction. Single molecule electronic devices offer exciting perspectives for further minituarization of electronic circuits with a potentially large impact in applications. Several experiments have demonstrated the possibility to attach individual molecules to leads and to

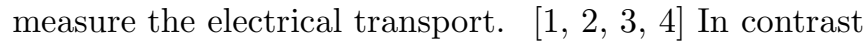
to single electron transistors (SETs) based on quantum dots [5] the electronic structure of molecular devices can be chemically 'designed' for specific applications. When the molecule is coupled weakly to the electrodes, i.e. via electron tunneling, charging effects, semi-classically determined by the small capacitance of the molecule, become important. The interplay of charging effects with the specific structure of the molecular orbitals leads to nontrivial current voltage $(I-V)$ characteristics [6, 7. Very recent experiments demonstrated both Coulomb blockade and the Kondo effect in three terminal transport through a single molecular level [8, 9.

Using benzene as a prototypical example we investigate novel effects that arise when transport through several competing electronic configurations becomes possible. We derive a semi-quantitative model for the conducting many-body states of the system from electronic structure calculations. For weak coupling to the electrode we compute transport within the golden rule approximation (sequential tunneling) and include screening of the applied electric field as well as radiative transitions between the electronic states of the molecule. We predict a current collapse in the $I-V$ curve and strong negative differential conductance (NDC) due to the occurrence of a "blocking" state when the molecule is coupled to the electrode at the para-position. For coupling at the metaposition, the $I-V$ curve displays a series of steps, but no NDC. We demonstrate how the specific spatial structure of the molecular orbitals qualitatively determines electronic transport. Finally, we discuss the limits of the model and the impact of disorder and symmetry breaking effects likely encountered in an experimental realization.
The Model. To perform transport calculations in the weakly coupled regime, we extract an effective model from electronic structure calculations of the molecule. For benzene transport is assumed to be dominated by the $\pi$-electron system, but generalizations are straightforward. We first perform Hartree-Fock calculations in a suitable basis and then transform the Hamiltonian to the molecular orbital basis. We then integrate out the $\sigma$ electrons of the system arriving at an effective interacting model Hamiltonian for the $\pi$ electrons of the system in the presence of the atomic cores and the "frozen" density of the $\sigma$ electrons:

$$
\mathcal{H}_{\pi}=\sum_{i j \sigma} \epsilon_{i j} c_{\sigma, i}^{\dagger} c_{\sigma, j}+\sum_{i j k l \sigma \sigma^{\prime}} U_{i j k l} c_{\sigma, i}^{\dagger} c_{\sigma^{\prime}, j}^{\dagger} c_{\sigma, k} c_{\sigma, l}
$$

The second quantized operators $c_{\sigma, i}^{\dagger}, c_{\sigma, i}$ create/destroy electrons of spin $\sigma$ in orthogonalized Wannier-like orbitals $\Phi_{i}$ centered at the carbon atoms. While this model neglects $\sigma-\pi$ mixing for certain excited states of the molecule 110, 11, its parameters account for the detailed electronic structure of the molecule. For the current work, we compute the model parameters only once, using an augmented double-zeta quality atomic natural orbital (ANO) basis set that was truncated to contain only one $2 \mathrm{p}$-shell for each carbon. When applying a bias over the molecule this neglects the higher order effect of field screening by $\sigma$ electrons and its impact on the $\pi$ electrons. Note that in our approach there is no over-counting of interaction terms like in "interaction enhanced" density functional treatments in solid state physics 12, 13].

We find that the low-energy spectrum of benzene obtained from the diagonalization of the effective Hamilton operator eq. 1 compares favorably with the spectrum directly obtained from accurate multi-reference configuration interaction calculations [11, 14]. The remaining differences can be understood by the lack of $\sigma-\pi$ mixing and do not qualitatively affect the transport properties. 
The restriction to a pure $\pi$-electron system is more severe for the charged states, as discussed in some detail below.

To account for the effect of an external bias potential $V^{e x t}$ on the electrons we include a term

$$
\mathcal{H}_{\text {bias }}=e \sum_{i j \sigma \sigma^{\prime}} V_{i j}^{e x t} c_{\sigma, i}^{\dagger} c_{\sigma, j}
$$

with $V_{i j}^{\text {ext }}=\int d \mathbf{r} \Phi_{i}(\mathbf{r}) V^{e x t}(\hat{\mathbf{r}}) \Phi_{j}(\mathbf{r})$ and $V^{e x t}(\hat{\mathbf{r}})=\left(V_{L}+\right.$ $\left.V_{R}\right) / 2-V_{\text {bias }}(\hat{\mathbf{r}} / L)$ in the Hamiltonian. $V_{L, R}$ is the chemical potential in the left/right electrode, distances are measured from the center of the molecule. The matrix elements $V_{i j}^{e x t}$ also result from the electronic structure calculation described above. We consider a bias $V_{\text {bias }}=V_{L}-V_{R}$ aligned with the transport direction ( $x$-direction). The parameter $L$ indicates the length over which the external bias falls off (we choose $L=0.4 \mathrm{~nm}$ ).

Diagonalization and Transport Calculation. To compute the transport properties of the system in the weak coupling limit we diagonalize the Hamiltonian eq. 1 in the appropriate charge/spin/symmetry sectors. For the current model, we have one "single-particle" state (Wannier state $\Phi_{i}$ ) per carbon site $i$, resulting in a total of $4^{N}=4096$ (with $N=6$ sites) many-body states $|s\rangle$. For the present model, charge, spin and symmetry adaptation reduce the size of effective Hamiltonian matrices sufficiently to permit their diagonalization with standard linear algebra packages. Care is needed to handle degeneracies properly. The energy degenerate states need to be additionally diagonalized to obtain states with integer (half-integer) total spin for states with even (odd) number of electrons. This also helps for the transport calculation, since the transition matrix elements can be summed over the magnetic quantum numbers, i.e. we only need to consider one representative of the degenerate spaces (we do not consider application of a magnetic field here).

After diagonalization of the Hamiltonian we have the many-body eigenstates $|s\rangle$ with the corresponding energies $E_{s}$ and their total spin $S_{s}$. We use a Master equation approach 7] for the occupation probabilities $P_{s}$ in a stationary state. The transition rates $\Sigma_{s s^{\prime}}$ from state $s^{\prime}$ to $s$ are computed in perturbation theory using the golden rule. The "perturbation" is the coupling of the molecule to the leads

$$
H_{\text {mol-leads }}=\left(\frac{\Gamma}{2 \pi \rho_{e}}\right)^{1 / 2} \sum_{\mathbf{k} \sigma \alpha l}\left(c_{l \sigma}^{\dagger} a_{\mathbf{k} \sigma \alpha}+\text { h.c. }\right)
$$

and (optionally) the coupling to electromagnetic fields (photons). $\Gamma$ is the coupling strength (in units of energy) of leads to the benzene and $\rho_{e}$ is the density of states of the electrons in the electrode (assumed constant). The operators $a_{\mathbf{k} \sigma \alpha}$ and their hermitian conjugates destroy/create electrons with momentum $\mathbf{k}$ and spin $\sigma$ in electrode $\alpha=$ left/right. For simplicity, we assume that tunneling is only possible through two "contact" carbon atoms which we choose to be at the 1 and 4 (para) positions unless noted otherwise.

As we do not consider the leads microscopically, the coupling of molecule states $|s\rangle$ is determined by the overall coupling strength $\Gamma$ and the relative wave function amplitude of the state $|s\rangle$ at the coupling carbon site $l$. For the transition rates we have $\Sigma_{s s^{\prime}}=\left(\sum_{\alpha, p= \pm} \Sigma_{s s^{\prime}}^{\alpha p}\right)+\Sigma_{s, s^{\prime}}^{d}$ where $\Sigma_{s s^{\prime}}^{\alpha p}$ is the tunneling rate to/from electrode $\alpha$ for creation $(p=+)$ or destruction $(p=-)$ of an electron on the molecule. We have

$$
\Sigma_{s s^{\prime}}^{\alpha+}=\Gamma f_{\alpha}\left(E_{s}-E_{s^{\prime}}\right) \sum_{\sigma}\left|\sum_{l}<s\right| c_{l \sigma}^{\dagger}\left|s^{\prime}>\right|^{2},
$$

and a corresponding equation for $\Sigma_{s^{\prime} s}^{\alpha-}$ by replacing $f_{\alpha} \rightarrow$ $1-f_{\alpha}$, where $f_{\alpha}$ is the Fermi function. When including relaxation by radiative transitions, we use the dipole approximation with dipole transition moments $\mathbf{d}_{i, j}=$ $\int d \mathbf{r} \Phi_{i}(\mathbf{r}) \hat{\mathbf{r}} \Phi_{j}(\mathbf{r})$ obtained from the electronic structure calculations. The corresponding transition rates are

$$
\Sigma_{s s^{\prime}}^{d}=\frac{4 e^{2}}{3 \hbar^{3} c^{3}}\left(E_{s}-E_{s^{\prime}}\right)^{3} N_{b}\left(E_{s}-E_{s^{\prime}}\right)|<s| \mathbf{d}\left|s^{\prime}>\right|^{2}
$$

where $N_{b}(E)$ denotes the equilibrium Bose function. Note that for emission $E_{s}-E_{s^{\prime}}$ is negative, and $N_{b}(-|E|)=-\left(1+N_{b}(|E|)\right)$.

The total transition matrix $\Sigma_{s s^{\prime}}$ consists of blocks connecting $N$ and $N \pm 1$ electron states (tunneling processes) and blocks from the radiative transitions that do not change the electron number on the molecule. Taking only one member of the subspace of spin and energy degenerate states into account, the rank $r$ of the transition matrix is 1716 . The stationarity condition $\dot{P}_{s}=0$ can be written as $\sum_{s^{\prime}} A_{s s^{\prime}} P_{s^{\prime}}=0$ with the matrix $A_{s s^{\prime}}=\Sigma_{s s^{\prime}}-\sum_{s^{\prime \prime}} \Sigma_{s^{\prime \prime} s} \delta_{s s^{\prime}}$. This implies that $A_{s s^{\prime}}$ has an eigenvector with zero eigenvalue, which is the wanted solution for $P_{s}$. Rather than computing this eigenvector by brute force, to speed up the calculation we make use of $\sum_{s} P_{s}=1$ to eliminate one row/column, thus reformulating the eigenvector problem into one of solving an inhomogeneous linear system of rank $r-1$. Eventually, the current in the left and right electrode is calculated via $I_{\alpha}=e \sum_{s, s^{\prime}}\left(\sum_{s s^{\prime}}^{\alpha+} P_{s^{\prime}}-\sum_{s^{\prime} s}^{\alpha-} P_{s}\right)$.

In this paper, we consider symmetric bias only. Applying an asymmetric bias would effectively imply different coupling to the left and right leads and has been previously discussed [7]. Finally, we note that we must have $\Gamma \ll k_{b} T \approx 40 \mathrm{meV}$ for perturbation theory to apply at room temperature $\mathrm{T}$.

Results: To elucidate the impact of various effects on the current we have performed transport calculations with and without radiative transitions (relaxation) and with and without effect of the applied bias.

On the left panel of Fig. 1 we present the $I-V$ curve obtained without the effect of the applied field 


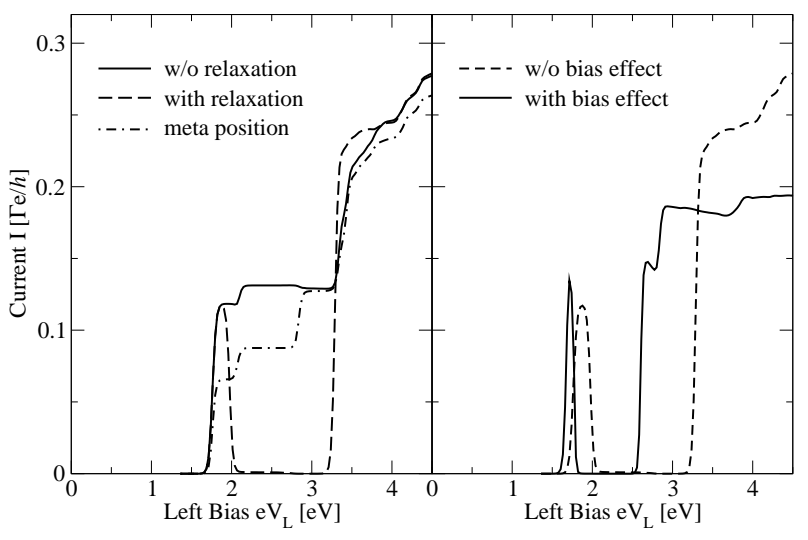

FIG. 1: $I-V$ characteristics for symmetric bias. Left Panel: $I-V$ curve without (solid line) and with radiative relaxation (dashed line) without inclusion of bias effect. The current collapses at a bias when the antisymmetric anion state can become occupied by radiative relaxation from an excited anion state. For coupling of the right electrode at the meta position (dash-dotted line) the "blocking state" has finite tunnel coupling to both electrodes and no current collapse is observed. Right Panel: With inclusion of the bias effect (solid line) the onset of current is generally shifted to lower bias, but the current collapse remains and additional weak NDC occurs at larger bias.

(zero bias electronic structure). Without radiative relaxation (solid line) the $I-V$ curve consists of a series of steps of which only a few are well resolved on this scale. The first step is associated with the population of the first $\pi^{*}$ orbital of molecule (molecular charge $=-e$ ), an electron hops onto the lowest available level and then hops off again. At slightly larger bias, a transition of the anion to the first excited state of the neutral molecule becomes possible, resulting in a slight increase of the current. If the bias is sufficiently large this excited state may now accept another electron to populate higher excited states of the anion or low-lying states of the di-anion, resulting in a rapidly growing cascade of transitions between literally hundreds of states of the system. In the model considered here the growth of this cascade leads to quasiohmic behavior above $3.6 \mathrm{eV}$. In our calculation the first states of the di-anion become occupied at about $4.5 \mathrm{eV}$.

The inclusion of radiative transitions has a dramatic effect on the $I-V$ curve (dashed line). We observe a collapse of the current over a substantial range of the applied bias (2.1-3.4 eV). The reason for this collapse is the population of a "blocking" state in the cascade of transitions that becomes possible when exited states of the neutral molecule and anion become accessible. Above approximately $2.1 \mathrm{eV}$ bias an excited state of the anion at about $5.6 \mathrm{eV}$ (see Fig. 2), becomes partially populated in the transport cascade. This state can decay by photon emission to either a symmetric or an antisymmetric many body state (with respect to the plane through the

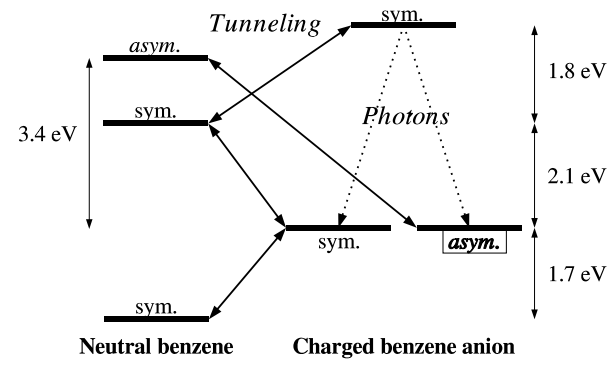

FIG. 2: Sketch of the energetics and symmetry of the relevant neutral and anion states. At a left bias of $2.1 \mathrm{eV}$ the antisymmetric blocking state becomes occupied via radiative relaxation. At $3.4 \mathrm{eV}$ electrons can escape the blocking state via tunneling to the antisymmetric state of neutral benzene.

transport axis and perpendicular to the molecular plane) of the anion. In the bias range of the current collapse this state cannot decay by coupling to the leads, because the lowest neutral states are symmetric (see Fig. 2) and the tunneling preserves the symmetry. Since there are no further radiative transitions possible on the molecule, the rate equations contain no draining term from this state. As a result, in the stationary state, the probability of occupying the "blocking" state is unity and the current ceases to flow. At a larger bias $(3.4 \mathrm{eV})$, the first escape channel opens, and the system can decay to the first antisymmetric excited state of the neutral molecule, which can then decay further by photon emission.

The above calculations simplify the illustration of the mechanism of NDC, because the energies of the participating states are independent of the applied bias. In the physical system the various states couple differently to the applied field. The solid line in the right panel of Fig. 1 shows the $I-V$ curve of the benzene with radiative relaxation and with the effect of the external potential applied parallel to the transport axis. We note a shift of the first step in the $I-V$ curve, which is due to differential screening effects between the ground state of the neutral molecule and the anion. At larger bias, the differential effects on all of the states in the cascade result in a significant renormalization of the $I-V$ curve. However, the current collapse still occurs, although the voltage window is reduced. The onset of quasi-ohmic behaviors occurs at higher voltage and a series of weak NDC effects occurs due to redistribution of occupation probabilities in favor of states with slightly smaller "transmission".

If we couple the right electrode to the meta position of benzene (left panel, dash-dotted curve) we find no current collapse with or without radiative relaxation or bias effect. This can be readily understood by the fact that at the meta position the wave function of the formerly blocking state is non-vanishing and electrons can tunnel out to the right electrode. Consequently, we observe a series of current steps similar (but not identical) to the 
case of coupling to the para position without relaxation.

Discussion: In the above we have described transport in an idealized, though semi-quantitative, $\pi$-electron model of weakly coupled benzene and found a dramatic suppression of the current in a finite voltage window. The mechanism for this effect is the occupation of a so-called blocking state of the molecule, which cannot decay for symmetry reasons. In the following we will summarily discuss its stability under the influence of a number of effects not considered in our calculation, but likely to be present in a experimental realization. Details will be discussed in a forthcoming publication [15].

(i) Transport through the $\sigma$-system: The ionization potential of benzene is rather high, for this reason no cationic states (with either $\sigma$ or $\pi$ holes) are relevant for the bias range considered here and attainable in experiment. However, for the anion, low lying Rydberg states are predicted in quantum chemical calculations with augmented basis sets containing diffuse functions. In these states the additional particle occupies an electron cloud of $\sigma$ symmetry that is smeared out over the entire molecule and has significant charge density in the center of the ring. Including these states, we expect the onset of current in the $I-V$ curve, i.e. the first current step, will be due to tunneling into the lowest Rydberg state, at about $1 \mathrm{eV}$. Nevertheless we believe that the NDC effect will be preserved. The current collapse is due to the competition between a slow process (cascade filling the blocking level) with an even slower one (decay of the blocking level). NDC occurs when probability density is redirected from "conducting" many-body states with large tunnel coupling to non-conducting many-body states even as the voltage increases. Note that the existence of a single "blocking path" is sufficient for NDC, irrespective of the number of competing non-blocking cascades.

The addition of Rydberg states below the blocking $\pi$ state of the anion permit its decay to symmetric or antisymmetric Rydberg states of the anion. Note that the spectrum of the neutral molecule is unaffected no additional low-lying configurations must be considered. Therefore a new radiative cascade on the anion ultimately leads to a symmetric Rydberg state, which can couple to the ground state configuration of the neutral molecule. The amplitude for this relaxation, however, is slow, because the transition dipole moment from the blocking $(\pi)$ configuration to the Rydberg $(\sigma)$ configuration is small [15] (involving a dipole transition moment perpendicular to the benzene plane).

(ii) Symmetry breaking: In most experimental realizations for two terminal transport, with the possible exception of STM contacts [16], the alignment of the molecule with respect to the electrodes is uncontrolled. It is therefore likely that the applied bias is not aligned with the transport direction. In this case the degenerate states of the anion mix, and transport is no longer completely blocked. However, according to the rate argument presented above, it is still impeded, and a reduced NDC remains observable. The same consideration holds true for slow vibrational modes of the molecule, like breathing and buckling [17. Fast vibrations can provide radiationless relaxation and should be considered at the quantum level. They can be particularly important if they generate transition matrix elements that allow for dipole forbidden electronic transitions in the undistorted system.

Summary: In weakly coupled molecules the concept of the "blocking" state suggests new opportunities for the design of single molecule electronic devices. In this investigation we have demonstrated how transport through several competing many-body states can result in strong NDC and current collapse over a significant voltage window. Our calculations predict the existence of this effect for a realistic molecule. Note that for $V_{\text {bias }}$ in the vicinity of the current peak, the application of a gate voltage $V_{g}$ may induce a large asymmetric change in the current, which would first rise and then drop again upon scanning of $V_{g}$ through a certain range. This behavior could be useful in various electronic devices.

Acknowledgments. One of us (M.R.W.) would like to thank Philip Stampfuß for assistance with the electronic structure calculations. WW acknowledges support of the DFG (grant WE 1863/10-1), the von-Neumann-Institute for Computing and the HLRZ Karlsruhe.

[1] M. A. Reed et al., Science 278, 252 (1997).

[2] C. Kergueris et al., Phys. Rev. B 59, 12505 (1999).

[3] J. Reichert et al, Phys. Rev. Lett. 88, 176804 (2002).

[4] M. Mayor et al, Angew. Chem. 114, 1228 (2002).

[5] 'Mesoscopic Electron Transport', eds. L.L. Sohn et al. (Kluwer 1997).

[6] J. Chen et al., Science 286, 1550 (1999).

[7] M. H. Hettler, H. Schoeller and W. Wenzel, Europhys. Lett. 57, 571 (2002); ibid. in Nano-Physics and BioElectronics: A New Odyssey, eds. T. Chakraborty, F. Peeters and U. Sivan (Elsevier 2002).

[8] J. Park, A. N. Pasupathy et.al., Nature 417, 722 (2002).

[9] W. Lian et.al., Nature 417, 725 (2002).

[10] B. O. Roos, K. Andersson and M. P. Fülscher, Chem. Phy. Lett. 192, 5 (1992).

[11] T. Hashimoto, H. Nakano, K. Hirao, J. Mol. Struct. 451, 25 (1998).

[12] M. M. Steiner, R. C. Albers and L. J. Sham, Phys. Rev. B45, 13272 (1992).

[13] K. Held et al. cond-mat/0112079 and references therein.

[14] P. Stampfuß and W. Wenzel, J. Comput. Chem. 20, 1559 (1999).

[15] M. R. Wegewijs, W. Wenzel, M. H. Hettler and H. Schoeller, in preparation.

[16] X. D. Cui et. al. Science 294, 571 (2001).

[17] M. Di Ventra, S. T. Pantelides and N. D. Lang, Phys. Rev. Lett. 88, 046801 (2002). 